\title{
DroidAnalytics: A Signature Based Analytic System to Collect, Extract, Analyze and Associate Android Malware
}

\author{
Min Zheng, Mingshen Sun, John C.S. Lui \\ Computer Science \& Engineering Department \\ The Chinese University of Hong Kong
}

\begin{abstract}
Smartphones and mobile devices are rapidly becoming indispensable devices for many users. Unfortunately, they also become fertile grounds for hackers to deploy malware and to spread virus. There is an urgent need to have a "security analytic \& forensic system" which can facilitate analysts to examine, dissect, associate and correlate large number of mobile applications. An effective analytic system needs to address the following questions: How to automatically collect and manage a high volume of mobile malware? How to analyze a zero-day suspicious application, and compare or associate it with existing malware families in the database? How to perform information retrieval so to reveal similar malicious logic with existing malware, and to quickly identify the new malicious code segment? In this paper, we present the design and implementation of DroidAnalytics, a signature based analytic system to automatically collect, manage, analyze and extract android malware. The system facilitates analysts to retrieve, associate and reveal malicious logics at the "opcode level". We demonstrate the efficacy of DroidAnalytics using 150,368 Android applications, and successfully determine 2,494 Android malware from 102 different families, with 342 of them being zero-day malware samples from six different families. To the best of our knowledge, this is the first reported case in showing such a large Android malware analysis/detection. The evaluation shows the DroidAnalytics is a valuable tool and is effective in analyzing malware repackaging and mutations.
\end{abstract}

\section{Introduction}

Smartphones are becoming prevailing devices for many people. Unfortunately, malware on smartphones is also increasing at an unprecedented rate. Android OS-based systems, being the most popular platform for mobile devices, have been a popular target for malware developers. As stated in [1], the exponential growth of mobile malware is mainly due to the ease of generating malware variants. Although there are number of works which focus on Android malware detection via permission leakage, it is equally important to design a system that can perform comprehensive malware analytics: analyze and dissect suspicious applications at the opcode level (instead at the permission level), and correlate these applications to existing malware in the database to determine whether they are mutated malware or even zero-day malware, and to discover which legitimate applications are infected.

Challenges: To realize an effective analytic system for Android mobile applications, we need to overcome several technical hurdles. First, how to systematically collect malware from the wild. As indicated in [2], new malware variants are always hidden in many different third-party markets. Due to the competition of anti-virus companies and their fear of accidentally releasing malware to the public, companies are usually reluctant to share their malware database to researchers. Researchers in academic can only obtain a small number of mobile malware samples. Hence, how to automate a systematic process to obtain these malicious applications is the first hurdle we need to overcome.

The second hurdle is how to identify repackaged applications (or mutated malware) from the vast ocean of applications and malware. As reported in [3], hackers can easily transform legitimate applications by injecting malicious logic or obfuscated program segments so that they have the same structure as the original application but contain malicious logic. Thus, how to determine whether an application is a repackaged or obfuscated malware, and which legitimate applications are infected is very challenging.

The third hurdle is how to associate malware with existing malware (or application) so as to facilitate security analysis. The existing approach of using cryptographic hash or package name as an identifier is not effective because hackers can easily change the hash value or package name. Currently, security analysts need to go through a laborous process of manually reverse engineer a malware to discover malicious functions and structure. There is an urgent need to have an efficient method to associate malware with other malware in the database, so to examine their commonalities at the opcode level.

Contributions: To address these problems mentioned above, we present the design and implementation of DroidAnalytics, an Andorid malware analytic system for malware collection, signature generation, information retrieval, and malware association based on similarity score. Furthermore, DroidAnalytic can efficiently detect zero-day repackaged malware. The contributions of our system are:

- DroidAnalytics automates the processes of malware collection, analysis and management. We have successfully collected 150,368 Android applications, and determined 2,494 malware samples from 102 families. Among those, there are 342 zero-day malware samples from six different malware families. We also plan to release the malware database to the research community (please refer to https://dl.dropbox.com/u/37123887/malware.pdf). 
- DroidAnalytics uses a multi-level signature algorithm to extract the malware feature based on their semantic meaning at the opcode level. This is far more robust than a cryptographic hash of the entire application. We show how to use DroidAnalytics to combat against malware which uses repackaging or code obfuscation, as well as how to analyze malware with dynamic payloads (see Sec. IIII).

- Unlike previous works which associate malware via "permission", DroidAnalytics associates malware and generates signatures at the app/class/method level. Hence, we can easily track and analyze mutation, derivatives, and generation of new malware. DroidAnalytics can reveal malicious behavior at the method level so to identify repackaged malware, and perform class association among malware/applications (see Sec. [V].

- We show how to use DroidAnalytics to detect zeroday repackaged malware. We have found 342 zero-day repackaged malware in six different families (see Sec. V).

\section{Design \& Implementation of DroidAnalytics}

Here, we present the design and implementation of DroidAnalytics. Our system consists of modules for automatic malware collection, signature generation, information retrieval and association, as well as similarity comparison between malware. We will also show how to use these functions to detect zero-day repackaged malware.

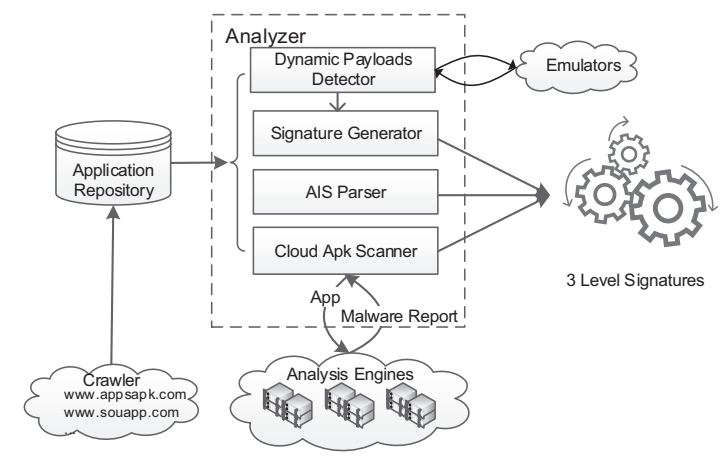

Fig. 1. The Architecture of the DroidAnalytics

\section{A. Building Blocks of DroidAnalytics}

Figure 1 depicts the architecture of DroidAnalytics and its components. Let us explain the design of each component.

- Extensible Crawler: In DroidAnalytics, we implement an application crawler based on Scrapy [4]. Users can specify official or third party market places, as well as blog sites and the crawler will perform regular mobile application download. The crawler enables us to systematically build up the mobile applications database for malware analysis and association. So far, we have collected 150,368 mobile applications and carried out detailed security analysis.

- Dynamic Payloads Detector: To deal with malware which dynamically downloads malicious codes via the Internet or attachment files, we have implemented the dynamic payloads detector component, which determines malicious trigger code within malware packages and tracks the downloaded application and its behavior in virtual machine. Firstly, it scans the package to find suspicious files such as .elf or .jar file. Hackers usually camouflage malicious files by changing their file type. To overcome this, this component scans all files and identifies files using their magic numbers instead of file extension. Secondly, if an application has any Internet behavior (e.g., Internet permission or re-delegating other applications to download files [5]), the dynamic payloads detector will treat these files as the target, then runs the application in the emulator. The system will use the forward symbolic execution technique to trigger the download behavior.Both the suspicious files within the package and dynamically downloaded files from the Internet will be sent to the signature generator (which we will shortly describe) for further analysis.

- Android App Information (AIS) Parser: AIS is a data structure within DroidAnalytics and it is used to represent . apk information structure. Using the AIS parser, analysts can reveal the cryptographic hash (or other basic signature) of an .apk file, its package name, permission information, broadcast receiver information and disassembled code, ..., etc. Our AIS parser decrypts the AndroidManifest.xml within an application and disassembles the .dex file into . smali code. Then it extracts package information from source code and retains it in AIS so analysts can easily retrieve this information.

- Signature Generator: Anti-virus companies usually use cryptographic hash, e.g., MD5, to generate a signature for an application. This has two major drawbacks. Firstly, hackers can easily mutate an application and change its cryptographic hash. Secondly, the cryptographic hash does not provide sufficient flexibility for security analysis. In DroidAnalytics, we use a three-level signature generation scheme to identify each application. This signature scheme is based on the mobile application, classes, methods, as well as malware's dynamic payloads (if any). Our signature generation is based on the following observation: For any functional application, it needs to invoke various Android API calls, and Android API calls sequence within a method is difficult to modify (unless one drastically changes the program's logic, but we did not find any from the 150,368 applications we collected that used this obfuscation technique). Hence, we generate a method's signature using the API call sequence, and given the signature of a method, create the signature of a class which composes of different methods. Finally, the signature of an application is composed of all signatures of its classes. We like to emphasize that our signature algorithm is not only for defense against malware obfuscation, but more importantly, facilitating malware analysis via class/method association (we will show in later sections). Let us present the detail of signature generation. (a) Android API calls table: Our system uses the API calls table of the Android SDK. The android.jar file is the framework package provided by the Android SDK. We use the Java reflection [6] to obtain all descriptions of the API calls. 
For each API, we extract both the class path and the method name. We assign each full path method a hex number as part of the ID. For the current version of DroidAnalytics, we extract 47,126 full path methods in the Android SDK 4.1 version as our API calls table. Table \ depicts a snapshot of API calls table, e.g., android/content/Intent;-><init> is assigned an ID 0x30291.

\begin{tabular}{|l|l|}
\hline Full Path Method & Method ID \\
\hline \hline android/accounts/Account;-><init> & 0x00001 \\
$\vdots$ & $\vdots$ \\
android/content/Intent; -><init> & 0x30291 \\
android/content/Intent;->toUri & 0x30292 \\
android/telephony/SmsManager;->getDefault & 0x39D53 \\
android/app/PendingIntent;->getBroadcast & 0xF3E91 \\
\hline
\end{tabular}

TABLE I

EXAMPLE OF THE ANDROID API CALLS TABLE AND ASSIGNED IDS

(b) Disassembling process: Each Android application is composed of different classes and each class is composed of different methods. To generate signatures for each class or method, DroidAnalytics first disassembles an .apk file, then takes the Dalvik opcodes of the . dex file and transforms them to methods and classes. Then DroidAnalytics uses the Android API calls table to generate signatures.

(c) Generate Lev3 signature (or method signature): The system first generates a signature for each method and we call this the Lev3 signature. Based on the Android API calls table, the system extracts the API call ID sequence as a string in each method, then hashes this string value to produce the method's signature. Figure 2 illustrates how to generate the Lev3 signature of a method which sends messages to another mobile phone. Figure 2 shows that the method contains three API calls. Using the Android API calls table (as in Table \), we determine their IDs. Signature of a method is generated by cancatenation of all these IDs. Note that DroidAnalytics will not extract the API calls which will not be executed in run time because these codes are usually generated via obfuscation. Furthermore, if a method (except the main method) will not be invoked by any other methods, signature generator will also ignore this method because this may be a defunct method generated by malware writers.

(d) Generate Lev2 signature (either class signature or dynamic payload signature): Next, DroidAnalytics proceeds to generate the Lev2 signature for each class, and it is based on the Lev3 signatures of methods within that class. Malware writers may use various obfuscation or repackaging techniques to change the calling order of the methods table in a . dex file. To overcome this problem, our signature generation algorithm will first sort the level 3 signatures within that class, and then concatenate all these level 3 signatures to form the level 2 signature.

Some malicious codes are dynamically downloaded from the Internet during execution. DroidAnalytics uses the dynamic payloads detector component to obtain the payload files. For

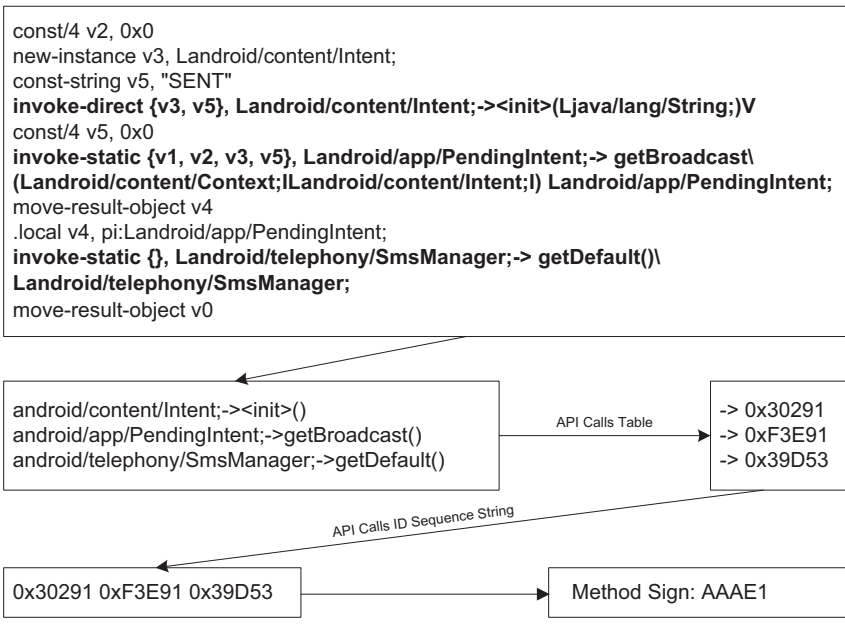

Fig. 2. The Process of Lev3 Signature Generation

the dynamic payloads which are .dex file or .jar file, DroidAnalytics treats them as classes within the malware. Given these files, the system checks their API call sequence and generates a Lev2 signature for each class within an application. For the dynamic payloads which contain, say, . elf file or . so file, DroidAnalytics treats them as a single class within that malware, then uses the cryptographic hash value (e.g., MD5) of the payload as its Lev2 signature. For the dynamic payloads which are .apk files, DroidAnalytics treats each as a new application and a class within the malware. DroidAnalytics first uses the cryptographic hash value (e.g., MD5) of the new . apk file as one Lev2 signature of that malware. Because the payload is a new application, DroidAnalytics will use the method we discussed to carry out a new signature generation.

(e) Generate Lev1 signature (or application signature): The Lev1 signature is based on the level 2 signatures, e.g., signatures of all qualified classes within an application. In addition, the signature generator will ignore the class (except the main class) which will not be invoked by any other classes since these defunct classes may be generated via obfuscation. Malware writers may use some repackaging or obfuscation techniques to change the order of the classes table of the . dex file, our signature algorithm will first sort all Lev2 signatures, then concatenate these Lev2 signatures to generate the Lev1 signature.

Figure 3 summarizes the framework of our signature algorithm. For example, the Lev3 signatures of $\mathrm{AAAE} 1$ and B2 $3 \mathrm{E} 8$ are the two method signatures within the same class. Based on these two (sorted) signatures, we generate the Lev2 signature of the corresponding class, which is 53EB3. Note that the Lev2 signature of C3EB3 is generated from a . dex file which is a dynamic payload used to execute the malicious behavior. Based on all sorted Lev2 signatures of all classes, we generate the Lev1 signature, F32DE, of the application.

For the current DroidAnalytics platform, we use a server which is of $2.80 \mathrm{GHz}$ Duo CPU processor, 4GB memory and 


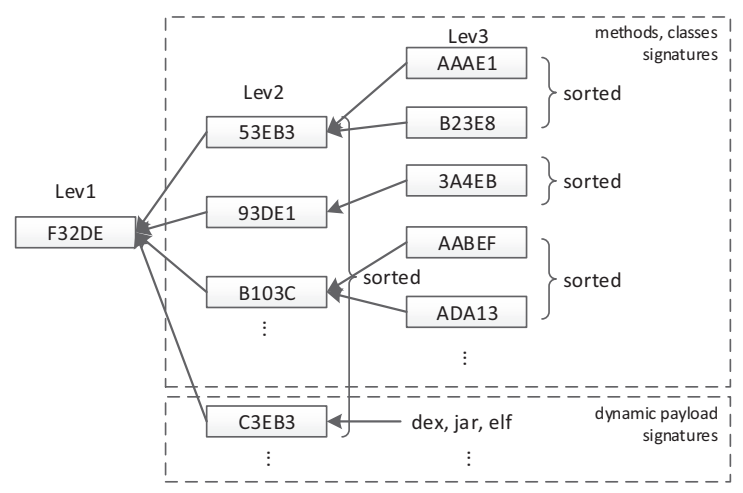

Fig. 3. Illustration of signature generation: the application (Lev1) signature, class level (Lev2) signatures and method level (Lev3) signatures.

2 TB hard disk, with two virtual machines in the server to implement the anti-virus engine. We carried out experiment to study the processing time to scanning and generating signatures. On average, it takes around 60 seconds to scan one application (includes the dynamic analysis), and around three seconds to generate all three level signatures, five seconds to generate AIS information, and one second to insert information into the database. As of November 2012, our system have downloaded 150,368 mobile applications from the following places: Google Play [7], nine Android third party markets (e.g., [8]-[10]), two malware forums [11], [12] and one mobile malware share blog [2]. The size of all downloaded application is $468 \mathrm{~GB}$.

\section{Utility \& Effectiveness of Signature Based System}

Here, we illustrate how DroidAnalytics' signatures can be used to analyze (and detect) malware repackaging, code obfuscation and malware with dynamic payloads.

A. Analyzing Malware Repackaging Repackaging obfuscation technique is commonly used by malware writers to change the cryptographic hash value of an .apk file without modifying the opcodes of the .dex file. This technique is different from the repackaged technique which is to inject new packages into the legitimate applications. For example, using Jarsigner utility of Java SDK to re-sign an .apk file only changes the signature part of one .apk file, and generates a new .apk file which preserves the same logic and functionality as the original one. Another example is using the Apktool [13], which is a reverse engineering tool to disassemble and rebuild an .apk file without changing any assembly code. Although there is no modification on the assembly codes, the recompiler may change the classes order and methods order during the recompiling process. Therefore, repackaging obfuscation is often used to mutate an existing malware to generate a new version with a different signature. If an anti-virus system only identifies malware based on a cryptographic hash signature, then repackaging obfuscation techniques can easily evade the detection.

DroidAnalytics can detect malware which is generated by repackaging obfuscation. Since there is no modification on the opcodes within the .dex file, and DroidAnalytics first sorts Lev2 and Lev3 signatures before generating the Lev1 signature. Therefore, DroidAnalytics will generate the same signature as the original even when one repackages the .apk file.

Experiment. To illustrate the above claim, we carry out the following experiment and Figure 4 illustrates the results. Opfake is a server-side polymorphism malware. The malware mutates automatically when it is downloaded. When analysts compare the cyclic redundancy codes (CRCs) of two Opfake downloads, it shows that the only meaningful change happens in the file data.db which is located in "res/raw/" folder. The modified data. $d b$ changes the signature data for the package in "META-INF" folder. By analyzing this form of malware, we find that all mutations in Opfake family happen in the same opcode (stored in classes.dex). Hence, our signature system will generate the same level 1 signature for all mutations in this malware family.

Figure 5 illustrates another example of using DroidAnalytics to analyze the Kmin family. We first calculate the Lev1 signatures of all 150,368 applications in our database. After the calculation, the result shows that the most frequent signature (which corresponds to the Lev1 signature 90b3d4af183e9f72f818c629b486fdec) comes from 117 files and all these files have different MD5 values. This shows that conventional cryptographic hashing (i.e., MD5) cannot identify malware variants but DroidAnalytics can effectively identify them. Also, these 117 files are all variants of the Kmin family. After further analysis, we discover that the Kmin family is a wallpaper changer application, and all its variants have the same application structure and same malicious behavior. The only difference is that they have different icons and wallpaper files.

B. Analyzing Malware which uses Code Obfuscation: A malware writer can use a disassembler (e.g., Apktool [13]) to convert a .dex file into .smali files, then injects new malware logic into the . smali code, rebuilds it back to a .dex file. Based on this rebuilt process, malware writers can apply various code obfuscation techniques while preserve the behavior as the original one in order to bypass the antivirus detection. As shown in [3], [14], many mobile anti-virus products are not effective to detect code obfuscated variants.

DroidAnalytics will not extract the API calls in methods and classes which will not be executed in run time (refer to Section III) because they are defunct and can be generated by obfuscators. In addition, the signature generation does not depend on the name of methods or classes, hence name obfuscation has no effect on our signature. Furthermore, the signature generation of DroidAnalytics is based on the analystdefined API calls table. So one can flexibly update the table entries to defend against various code obfuscation techniques. Experiment. To illustrate the effectiveness of DroidAnalytics against code obfuscation, we chose 30 different malware from three different families (10 samples in each family) and Table III illustrates our results. The malware families in our study are: Basebrid (or Basebridge), Gold-Dream, and Kungfu. We 


\section{Lev1 Signature Details of Opfake}

\begin{tabular}{|l|l|l|l|}
\hline MD5 & Package Name & Lev1 Signature & Detection Info \\
\hline e70761dd3d89d26eed3932d54089a121 & com.fywork.xiaohun & ec6618fc8b7de05d4b036dfd82e9a317 & Trojan-SMS.AndroidOS.Opfake.bo \\
\hline db9ae068d042a92c01fa5a26e3d434c8 & com.fywork.xiaohun & ec6618fc8b7de05d4bb036dfd82e9a317 & Trojan-SMS.AndroidOS.Opfake.bo \\
\hline da697083b250dd807671130f6e966a5d & com.fywork.xiaohun & ec6618fc8b7de05d4b036dfd82e9a317 & Trojan-SMS.AndroidOS.Opfake.bo \\
\hline e42b7361d15a29edce370372c2852e41 & com.fywork.xiaohun & ec6618fc8b7de05d4b036dfd82e9a317 & Trojan-SMS.AndroidOS.Opfake.bo \\
\hline d6926a52ad43d05e0982991a5634bf32 & com.fywork.xiaohun & ec6618fc8b7de05d4b036dfd82e9a317 & Trojan-SMS.AndroidOS.Opfake.bo \\
\hline
\end{tabular}

Fig. 4. Screen Capture of Opfake Family Lev1 Signature

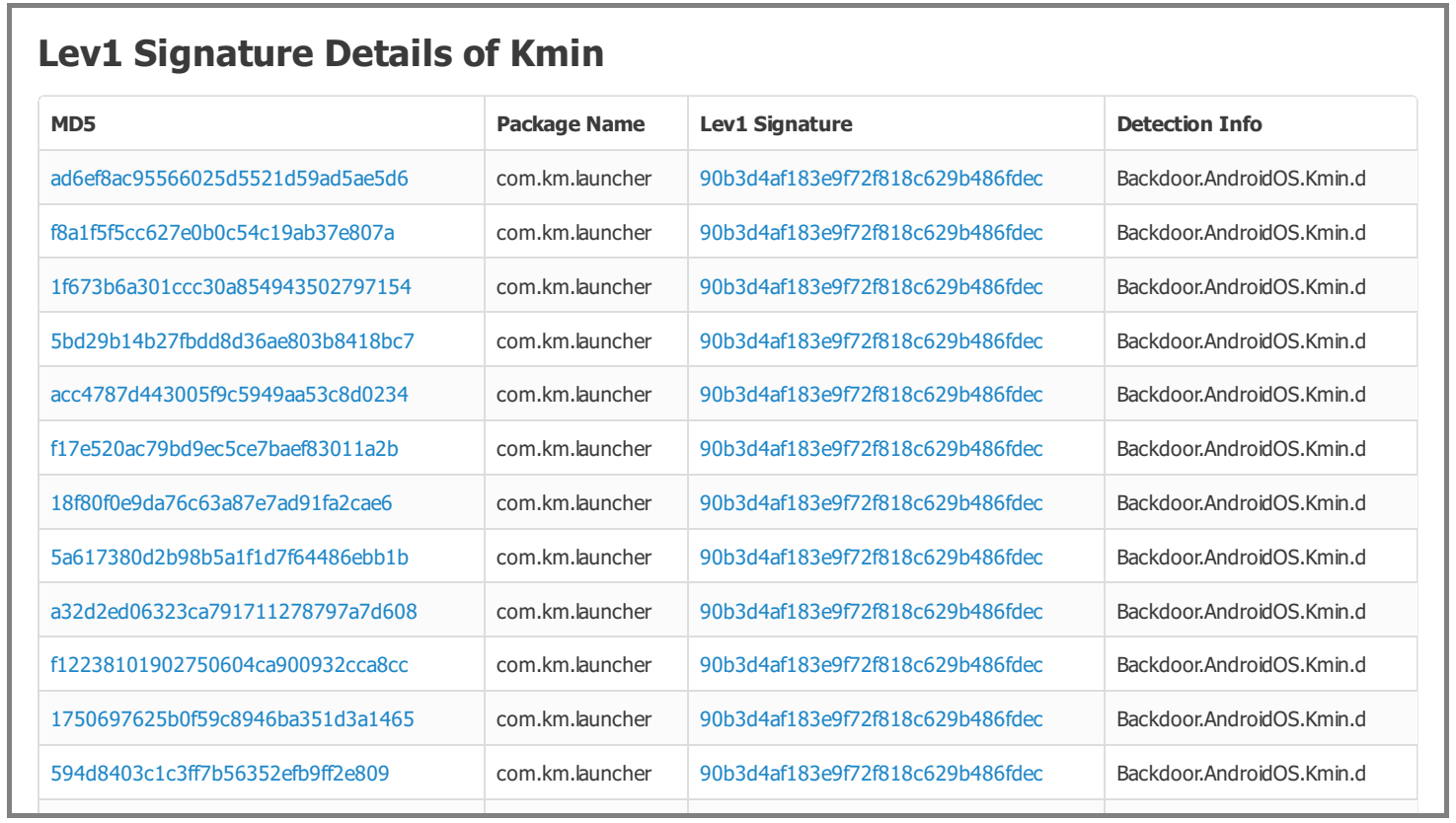

Fig. 5. Screen Capture of Kmin Family Lev1 Signature

use ADAM [15], a system which can automatically transform an original malware sample to different variants by various repackaging and obfuscation techniques (e.g. inserting defunct methods, modifying methods name, ...etc). We generate seven different variants for each malware. Then we put these 240 new malware samples into the DroidAnalytics system and check their signatures. After our signature calculations, the result shows that for each malware, the original sample and seven mutated variants have distinct MD5 hash values (3 repackaging, 4 code obfuscation), but all of them have the same level 1 signature. This shows that DroidAnalytics' signature system is effective in defending against code obfuscation.

C. Analyzing Malware with Attachement Files or Dynamic Payloads: Some malware will dynamically download file which contains the malicious code from the Internet. Also, some attachment files within a package may contain malicious logic but they can be concealed as other valid documents (e.g., . png file, .wma file). DroidAnalytics will treat these files as dynamic payloads. By using both static and dynamic analysis techniques described in Section III. DroidAnalytics accesses these payloads and generates different signatures.

Experiment. We carried out the following experiment. From our malware database, we used our signature system and detected some malware contain the same file with a .png filename extension. But when we check the magic number of this file, it is actually an .elf file. Upon further analysis, we found that this file is a root exploit and this malware belongs to the GinMaster (or GingerMaster) family. Another example is the Plankton family. By using dynamic analysis, DroidAnalytics discovered that all malware in this family will download a plankton_v0.0.4.jar (or similar .jar) when the main activity of the application starts. Further analysis revealed the . jar file contains malicious behavior, i.e., stealing browser's history information, making screen shortcuts and botnet logic. Table III depicts DroidAnalytics system detects some representative malware using dynamic 


\begin{tabular}{|l|l|l|l|l|}
\hline \hline MD5 & Pakage Name & Lev1 Signature & Malware Family & Description \\
\hline f7967f71b2f32287660ae9a3fa366022 & com.tutusw.phonespeedup & 3c83ed0f80646c8ba112cb8535c293dd & Kungfu & Original malware \\
f2e2d727f95fa868fd7ff54459e766e3 & com.tutusw.phonespeedup & 3c83ed0f80646c8ba112cb8535c293dd & Kungfu & Repackaging \\
e01f573cca83fdf2737be6ecee35fe33 & com.tutusw.phonespeedup & 3c83ed0f80646c8ba112cb8535c293dd & Kungfu & Repackaging \\
d383ceeb9c6ffcff8c0dd12b73ec43e3 & com.tutusw.phonespeedup & 3c83ed0f80646c8ba112cb8535c293dd & Kungfu & Repackaging \\
cd040541693693faca9fec646e12e7e6 & com.tutusw.phonespeedup & 3c83ed0f80646c8ba112cb8535c293dd & Kungfu & Obfuscation \\
401952d745cd7ca5281a7f08d3e2eede & com.tutusw.phonespeedup & 3c83ed0f80646c8ba112cb8535c293dd & Kungfu & Obfuscation \\
271c3965c7822ebf944feb8bbd1cfe7f & com.tutusw.phonespeedup & 3c83ed0f80646c8ba112cb8535c293dd & Kungfu & Obfuscation \\
8b12ccdc8a69cf2d6a7e6c00f698aaaa & com.tutusw.phonespeedup & 3c83ed0f80646c8ba112cb8535c293dd & Kungfu & Obfuscation \\
\hline \hline
\end{tabular}

TABLE II

EXAMPLES OF CODE OBFUSCATION

\begin{tabular}{|l|l|l|l|}
\hline \hline MD5 & Dynamic Payload & Description & Malware Family \\
\hline 34cb03276e426f8d61e782b8435d3147 & /assets/runme.png & ELF file to expoit root & GinMaster \\
a24d2ae57c3cee1cf3298c856a917100 & $\begin{array}{l}\text { /assets/gbfm.png } \\
\text { /assets/install.png } \\
\text { /assets/installsoft.png }\end{array}$ & ELF file to expoit root & GinMaster \\
& $\begin{array}{l}\text { /assets/runme.png } \\
\text { /assets/gbfa.png } \\
\text { 9e847c9a27dc9898825f466ea00dac81 } \\
\text { /assets/install.png } \\
\text { plankton_v0.0.4.jar }\end{array}$ & ELF file to expoit root & GinMaster \\
dcbe11e5f3b82ce891b793ea40e4975e & download in runtime & Plankton \\
\hline \hline
\end{tabular}

TABLE III

Examples of DyNamic Payloads

payloads.

\section{Analytic Capability of DroidAnalytics}

We conduct three experiments and show how analysts can study malware, carry out similarity measurement between applications, as well as perform class association among 150,368 mobile applications in the database.

A. Detailed Analysis on Malware: Using DroidAnalytics, analysts can also discover which class or method uses suspicious API calls via the permission recursion technique.

- Common Analytics on Malware. First, using the AIS parser, DroidAnalytics can reveal basic information of an application like the cryptographic hash (i.e., MD5 value), package name, broadcast receiver, ..., etc. This is illustrated in Figure 6 In addition, DroidAnalytics has a built-in cloudbased APK scanner that supports diverse anti-virus scan results (e.g., Kaspersky and Antiy) to help analysts for reference. Our cloud-based APK scanner is extensible to accommodate other anti-virus scan engines. Last but not least, DroidAnalytics can disassemble the .dex file and extract class number, method number, and API calls number in each application, class or method. These functionalities are useful so analysts can quickly zoom in to the meta-information of a suspicious malware. Figure 7 shows these functionalities.

- Permission Recursion. Current state-of-the-art systems examine the AndroidManifest. xml to discover permissions of an application. This is not informative enough since analysts do not know which class or which method uses these permissions for suspicious activities. In DroidAnalytics, we can discover the permission within a class or a method. Since each permission is related to some API calls [16].
In DroidAnalytics, we tag permission to API calls in each method. We combine the method permissions within the same class as class permission, and combine all class permissions as application permission. This helps analysts to quickly discover suspicious methods or classes.

- Experiment. In this experiment, we choose a popular malware family Kungfu and examine the permissions at the application/class/method levels. Malware in the Kungfu family can obtain user's information such as IMEI number, phone model,..., etc. It can also exploit the device and gain root privilege access. Once the malware obtained the root level access, it installs malicious application in the background as a back-door service.

We use DroidAnalytics to generate all three-level signatures. Figure 8 shows the partial structure of the signatures with permission recursion of two Kungfu malware: $A 4 E 39 D$ and D2EF 8A, and together with a legitimate application BEDIE3 (Lev1 signature). Firstly, based on the malware reports by our cloud APK scanner, we identify A4E39D and D2EF 8A are malware which come from the Kungfu family with different package names, net.atools.android.cmwrap and com.atools.netTrafficstats respectively. By analyzing the Lev2 signature, we discover that BCEED is the common class which is within the two Kungfu applications. Secondly, from the Lev3 signature of BCEED, we use the permission recursion method to expose the method $6 \mathrm{~F} 100$ with the INSTALL_PACKAGES permission. Note that the INSTALL_PACKAGES permission is a system permission which the application can install other unrelated applications. This shows how DroidAnalytics helps analysts to quickly discover the malicious code of methods and classes of the 


\section{Details}

This is a malware!

File Name: cab6872bddc4bf85e10fcf33e327d9ff.apk

Package: com.mybooft.myclips

Min Sdk Version: 2.3

Submit Time: 2012-05-22 21:40:15

Permissions(10):

android.permission.RECEIVE_BOOT_COMPLETED

android.permission.INTERNET

android.permission.READ_PHONE_STATE

android.permission.ACCESS_COARSE_LOCATION

android.permission.ACCESS_FINE_LOCATION

android.permission.INTERNET

android.permission.RECEIVE_SMS

android.permission.SEND_SMS

android.permission.INSTALL_PACKAGES

android.permission.WRTE_EXTERNAL_STORAGE

Receiver Intent(3):

android.intent.action.BOOT_COMPLETED

android.intent.action.SIG_STR

android.provider.Telephony.SMS_RECEIVED

Scan Results:

Kapersky: Detected HEUR_Trojan-Spy.AndroidOS.Adrd.a

Antiy: Pass

DroidAnalytics

Fig. 6. Screen Capture of Common Analytics on Malware

Kungfu family.

- Similarity Measurement. DroidAnalytics can also calculate the similarity between two Android mobile applications, and the computation is based on the three level signatures that we discussed. By comparing the similarity of applications, we can determine whether an application is repackaged malware. Moreover, because we use Lev2 (class) signature as the basic building block, our approach not only can provide the similarity scores between two applications, but can inform analysts the common and different code segments between two applications. This great facilitates analysts to carry out detailed analysis.

Our similarity score is based on the Lev2 signatures which represent all classes within an application. The similarity score is based on the Jaccard similarity coefficient. Given $f_{a}$ and $f_{b}$ as two Lev2 signature sets of two applications $a$ and $b$ respectively, $f_{a} \cap f_{b}$ refers to the same Lev2 signatures of $a$ and $b$ (or the common classes of these two applications), while $f_{a} \cup$ $f_{b}$ represents the set of classes of these two applications. $S(x)$ is a function which returns the total number of API calls in the set $x$. Our similarity score equation between two applications is:

$$
J_{\text {app }}\left(f_{a}, f_{b}\right)=\frac{S\left(f_{a} \cap f_{b}\right)}{S\left(f_{a} \cup f_{b}\right)} .
$$

Let us how to use this similarity score to carry out analysis. First, given a legitimate application $X$, we can find all applications $\left\{Y_{1}, Y_{2}, \ldots, Y_{k}\right\}$ which are the top $p \%$ (say $p=20$ ) applications that are similar to $X$. Second, in the procedure of calculating similarity, we can identify the common and different Lev2 signatures. As a result, repackaged or mutated malware (i.e., $Y_{i}$ ), can be easily detected using the similarity score and the classes of malicious behavior can be easily determined.

- Experiment. We carry out the experiment using similarity measurement based on the Lev2 and Lev3 signatures. We use a benign application and calculate the similarity with other applications in our database to find all repackaged malware of this benign application. Furthermore, we can discover the differences between this benign application and repackaged malware (at the code level) to see what malicious codes are repackaged into legitimate application.

We choose a legitimate application called "Touch alarm" 


\begin{tabular}{|c|c|c|c|}
\hline \multicolumn{4}{|l|}{ Lev2 Signature Details } \\
\hline Lev2 Signature & Class Path & $\begin{array}{l}\text { System } \\
\text { Call \# }\end{array}$ & Permission \\
\hline ddd52aa834fb01d43d40ecc8e8446691 & com/android/main/a.class & 1 & \\
\hline $796 c d 90585229 b c f 6 f c 92016787 c a f 76$ & com/android/main/b.class & 41 & android.permission.INTERNET \\
\hline 0611c13b8a800d835916f81e9727ebb7 & com/android/main/c.class & 12 & \\
\hline d574f3e9f167895ea4f97705774492b8 & com/android/main/SmsReceiver.class & 29 & android.permission.VIBRATE \\
\hline 9362e2688517f158b718aea04e148eea & com/android/main/TANCActivity.class & 14 & android.permission.ACCESS_NETWORK_STATE \\
\hline 8 ea570fd10f7502b0ca9418054f9e7bf & com/android/main/d.class & 85 & \\
\hline 4cf753b32156dd83d201fefeb52fe6ae & com/android/main/e.class & 41 & \\
\hline 37da78ccc56ebe428b7b9a65e43c9412 & com/android/main/f.class & 12 & \\
\hline b55be0a4f4bf133fd8f6ff8f275478bf & com/android/main/g.class & 4 & android.permission.ACCESS_NETWORK_STATE \\
\hline 5c660fad2e99b77de8f8a500287154b5 & org/jiaxxhaha/netraffic/ClearPreference.class & 36 & \\
\hline d4552bc5d9331411c638fb5722ccec03 & org/jiaxxhaha/netraffic/DetailActivity.class & 13 & \\
\hline 21f380e5b34827a8940b715735e9c19a & org/jiaxxhaha/netraffic/ConnectionActivity.class & 5 & \\
\hline
\end{tabular}

Fig. 7. Screen Capture of Detailed Lev2 Signature

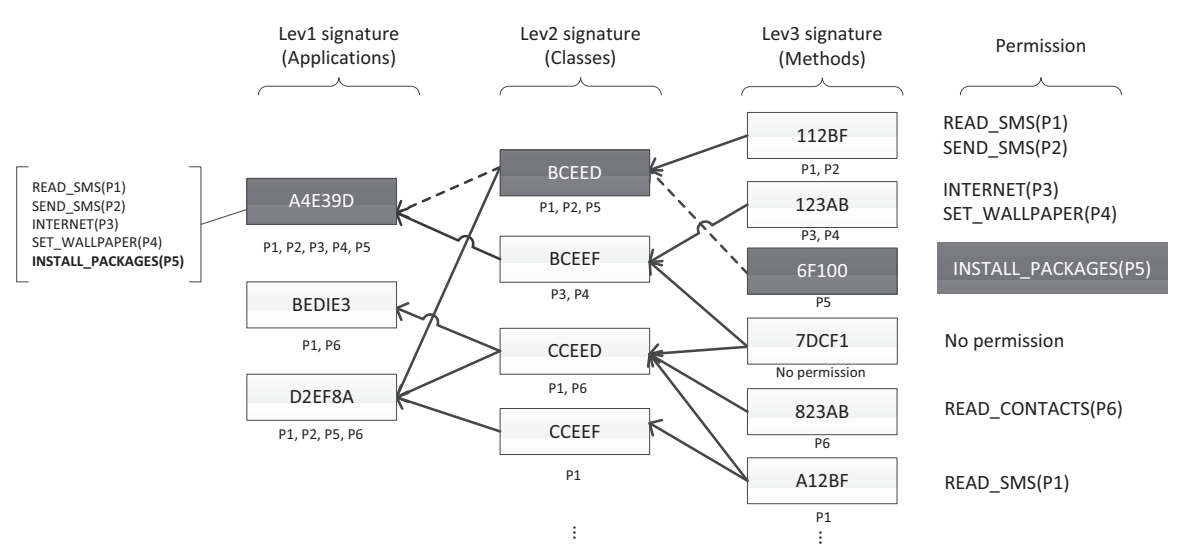

Fig. 8. Illustration of Detail Analytics on Malware

which is downloaded from Google official market. We use DroidAnalytics to compute the similar scores with malware in the database, Table IV shows the similarity scores between "Touch alarm" and other applications in our database (in decreasing order of similarity). From the table, we can clearly observe that the package names of the top six applications are the same: com.k99k.keel.touch.alert.freeze. The first one in the table is the legitimate "Touch alarm" application, while the following five applications are repackaged malware of Adrd. Adrd steals the personal information like IMEI, hardware information of users, and it will encrypt the stolen information and upload to some remote server. Moreover, it may dynamically download the newest version of Adrd and update itself. The table shows that the malware writer inserted malicious codes in benign "Touch alarm" and repackaged it to different variants of Adrd.

By using similarity measurement based on the three-level signature, DroidAnalytics reveals the difference between two applications at the code level. Table $\mathrm{V}$ shows the comparison of legitimate application "Touch alarm" and repackaged malware. Highlighted rows are the different level 2 signatures of the two applications, while the other rows represent same signature of common classes. This shows that DroidAnalytics can easily identify different classes of the two applications. By using permission recursion which we discussed previously, analysts can discover that the different level 


\begin{tabular}{|c|c|c|c|c|}
\hline MD5 & Package Name & $S\left(f_{a} \cap f_{b}\right) / S\left(f_{a} \cup f_{b}\right)$ & Similarity Score & Detection Result \\
\hline 278859faa5906bedb81d9e204283153f & com.k99k.keel.touch.alert.freeze & N/A & 1 & Not a Malware \\
\hline effb $70 \mathrm{ccb} 47 \mathrm{e} 8148 \mathrm{~b} 010675 \mathrm{ad} 870 \mathrm{c} 053$ & com.k99k.keel.touch.alert.freeze & $674 / 878$ & 0.76 & Adrd.w \\
\hline ef 46 ed2998ee 540 f96aaa1676993acca & com.k99k.keel.touch.alert.freeze & $674 / 878$ & 0.76 & Adrd.w \\
\hline cd 6 f 6 beff $21 d 4$ fe 5 caa 69 fb 9 ff 54 b 2 c 1 & com.k99k.keel.touch.alert.freeze & $674 / 878$ & 0.76 & Adrd.w \\
\hline $99 f 4111 \mathrm{a} 1746940476 \mathrm{e} 6 \mathrm{eb} 4350 \mathrm{~d} 242 \mathrm{f} 2$ & com.k99k.keel.touch.alert.freeze & $674 / 884$ & 0.77 & Adrd.a \\
\hline $49 b b f a 29 c 9 a 109 f f f 7 f e f 1 a a 5405 b 47 b$ & com.k99k.keel.touch.alert.freeze & $674 / 884$ & 0.77 & Adrd.a \\
\hline 39 ef06ad651c2acc290c05e4d1129d9b & org.nwhy.WhackAMole & $332 / 674$ & 0.49 & Adrd.cw \\
\hline & . & : & & \\
\hline
\end{tabular}

TABLE IV

SIMILARITY SCORES OF "TOuch Alarm" AND OTHER APPLICATIONS

2 signature e48040acb2d761fedfa0e9786dd2f3c2 has READ_PHONE_STATE, READ_CONTACTS and SEND_SMS in repackaged malware. Using DroidAnalytics for further analysis, we find suspicious API calls like android/telephony/TelephonyManager; -> getSimSerialNumber, android/telephony/gsm/ SmsManager;->endTextMessage and android/telephony/TelephonyManager;-> getDeviceID. Last but not least, we determine the malware writer inserted these malicious codes into legitimate application and published repackaged malware to various third party markets.

- Class Association. Traditional analysis on malware only focuses on one malware but can not associate malware with other malware or applications. DroidAnalytics can associate legitimate applications and other malware in the class level and/or method level. Given a class signature (or Lev2 signature), DroidAnalytics keeps track of how many legitimate applications or malware using this particular class. Also, with the methodology of permission recursion, DroidAnalytics can indicate the permission usage of this class signature. By using class association, we can easily determine which class or method may possess malicious behavior, and which class is used for common task, say for pushing advertisement. Lastly, for class signatures which are used by many known malware but zero legitimate application, these are classes that analysts need to pay special attention to because it is very likely that they contain malicious code and are used in many repackaged or obfuscated malware.

- Experiment. Using DroidAnalytics, we carry out the class association experiment using 1,000 legitimate appliactions and 1,000 malware as reported by Kaspersky. Figure 9 illustrates the results. After the class association, we discover a class (Lev2) signature $2 \mathrm{bcb} 4 \mathrm{f} 8940 \mathrm{f} 00 \mathrm{fb} 7 \mathrm{f50731ee} 341003 \mathrm{df}$ which is used by 143 malware and zero legitimate application. In addition, the 143 malware are all from the Geinimi family. Furthermore, this class has 47 API calls and uses READ_CONTACTS and SEND_SMS permissions. Therefore, we quickly identify this class contains malicious codes. In the signature database, we also find a class (Lev2) signature $9067 f 7292650 b a 0 b 5 c 725165111$ ef 04 e which is used by 80 legitimate applications and 42 malware. Further analysis shows that this class is used by similar number of legitimate applications and malware, and this class uses an advertisement library called DOMOB [17]. Another class (Lev2) signature a 007 d9e 3754 daef 90 ded300190903451 is used by 105 legitimate applications and 80 malware. Further examination shows that it is a class from the Google official library called AdMob [18]. This is illustrated in Figure 10.

In summary, all experimented samples are presented in Table VI which represents the known malware in our database. The detection results is based on the cloud anti-virus engine using various detection engines (i.e., Kaspersky and Antiy(linux version)). Note that in the last column, $R(G)$ represents repackaged (generic) malware family. For those malware families with less than five samples, we lump them as "others" in the table. Antiy (linux version) [19] is a commercial anti-virus product that we obtained from the company, and the product is known to run the same engine for detecting malware in smartphones. The rows are sorted in alphabetical order. Highlighted rows show the common malware families detected by both Kaspersky and Antiy. Others are uniquely detected by one anti-virus product. The penultimate row shows there are 1,295 common malware samples detected by these two anti-virus products. Hence, the number of unique malware samples is 2,148 .

\section{Zero-day Malware Detection}

Here, we show a novel methodology in using DroidAnalytics to detect the zero-day repackaged malware. We analyze three zero-day malware families to illustrate the effectiveness of our system.

\section{A. Zero-day Malware}

Zero-day malware is a new malware that current commercial anti-virus systems cannot detect. Anti-virus software usually relies on signatures to identify malware. However, signature can only be generated when samples are obtained. It is always a challenge for anti-virus companies to detect the zero-day malware, then update their malware detection engines as quickly as possible.

In this paper, we define an application as a zero-day malware if it has malicious behavior and it cannot be detected by popular anti-virus software (e.g., Kaspersky, NOD32, Norton) using their latest signature database. As of November, 2012, 


\begin{tabular}{|c|c|c|c|c|c|}
\hline \multicolumn{3}{|c|}{ Application A (MD5: $278859 \mathrm{faa} 5906 \mathrm{bedb} 81 \mathrm{~d} 9 \mathrm{e} 204283153 \mathrm{f})$} & \multicolumn{3}{|c|}{ Repackaged Malware of A (MD5: ef fb 70ccb47e8148b010675ad870c053) } \\
\hline Level 2 Signature & \# of API calls & Permission & Level 2 Signature & \# of API calls & Permission \\
\hline $02 \mathrm{bcaaa} 836 \mathrm{~d} 530035 \mathrm{bb} 8 \mathrm{db} 801 \mathrm{c} 85 \mathrm{cffd}$ & 17 & $\mathrm{~N} / \mathrm{A}$ & 02bcaaa836d530035bb8db801c85cffd & 17 & N/A \\
\hline \multirow[t]{2}{*}{$0611 \mathrm{c} 13 \mathrm{~b} 8 \mathrm{a} 800 \mathrm{~d} 835916 \mathrm{f} 81 \mathrm{e} 9727 \mathrm{ebb} 7$} & 12 & $\mathrm{~N} / \mathrm{A}$ & $0611 \mathrm{c} 13 \mathrm{~b} 8 \mathrm{a} 800 \mathrm{~d} 835916 f 81 \mathrm{e} 9727 \mathrm{ebb} 7$ & 12 & N/A \\
\hline & & & $178 \mathrm{e} 2481067 \mathrm{~b} 194 \mathrm{e} 00187 \mathrm{cfaadb} 12 \mathrm{f}$ & 1 & N/A \\
\hline \multirow[t]{4}{*}{$6720117047 d 39 c 2 e 2 e 90 f a 7 e 896 e 1615$} & 22 & WAKE_LOCK & $6720117047 \mathrm{~d} 39 \mathrm{c} 2 \mathrm{e} 2 \mathrm{e} 90 \mathrm{fa} 7 \mathrm{e} 896 \mathrm{e} 1615$ & 22 & WAKE_LOCK \\
\hline & & & e $48040 a c b 2 d 761$ fedfa $0 e 9786 d d 2 f 3 c$ & 62 & $\begin{array}{l}\text { READ_PHONE_STATE } \\
\text { READ_CONTACTS } \\
\text { SEND_SMS }\end{array}$ \\
\hline & . & & & . & \\
\hline & . & & : & : & : \\
\hline
\end{tabular}

TABLE V

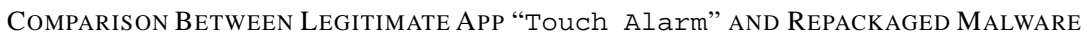

\section{Lev2 Signature Details}

\begin{tabular}{|c|c|c|c|c|c|}
\hline Lev2 Signature & Class Path & Legitimate Apps \# & Malware \# & API call \# & Malware Family \# \\
\hline 2bcb4f8940f00fb7f50731ee341003df & com/geinimi/c/i.class & 0 & 143 & 47 & 3 \\
\hline \multicolumn{6}{|l|}{ Details } \\
\hline Lev3 signature & \multicolumn{2}{|l|}{ Methods } & API call \# & \multicolumn{2}{|c|}{ Permissions } \\
\hline ce2c506e6614e4a78e837e6e92de0cf0 & \multicolumn{2}{|c|}{$\begin{array}{l}\text { android/content/Intent; ->() } \\
\text { android/app/PendingIntent; -> getActivity() } \\
\text { android/telephony/SmsManager;-> getDefault() } \\
\text { android/telephony/SmsManager; -> sendTextMessage() }\end{array}$} & 4 & \multicolumn{2}{|c|}{ android.permission.SEND_SMS } \\
\hline 1218be7ec4bbf26a481ef7145b043fab & \multicolumn{2}{|c|}{ 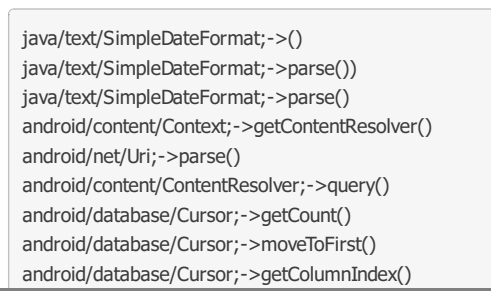 } & 43 & \multicolumn{2}{|c|}{$\begin{array}{l}\text { android.permission.SEND_SMS } \\
\text { android.permission.READ_CONTACTS }\end{array}$} \\
\hline
\end{tabular}

Fig. 9. Detailed Lev2 Signature of Repackaged Geinimi Malware

we use DroidAnalytics and have successfully detected 342 zero-day repackaged malware in five different families: AisRs, Aseiei, AIProvider, G3app, GSmstracker and YFontmaster (please refer to Table VII for reference). In this paper, we use the name of the injected package (not the name of the repackaged applications) as the name of its malware family. Furthermore, all samples are scanned by Kaspersky, NOD32, Norton and Antiy using their latest database in November, 2012. We also uploaded these samples to the virustotal [20] for malware detection analysis. Note that none of the submitted samples was reported as a malware by these engines when we carried out our experiments.

In [21], [22], authors reported that nearly $86.0 \%$ of all Android malware are actually repackaged versions of some legitimate applications. By camouflaging to some legitimate applications, repackaged malware can easily deceive users. Given the large percentage of repackaged malware, we explore the effectiveness of using DroidAnalytics to detect the zeroday repackaged malware.

\section{B. Zero-Day Malware Detection Methodology}

The process of detecting zero-day repackaged malware can be summarized by the following steps.

Step 1: We first construct a white list for common and legitimate classes. For example, we add all legitimate level 2 signatures, such as those in utility libraries (e.g., Json library) or advertisement libraries (e.g., Google Admob library, Airpush library) to the white list. All level 2 signatures in the white list will not be used to calculate the similarity score between two applications.

Step 2: We calculate the number of common API calls between two given applications in the database. This can be achieved by using the similarity score in Equation (2).

$$
S_{\text {app }}\left(f_{a}, f_{b}\right)=S\left(f_{a} \cap f_{b}\right),
$$

where $f_{a}$ and $f_{b}$ as two level 2 signature sets of two applications $a$ and $b$ respectively, $S(x)$ is a function to indicate the total number of API calls in the set $x$. The above similarity score between two repackaged malwares focus on the com- 


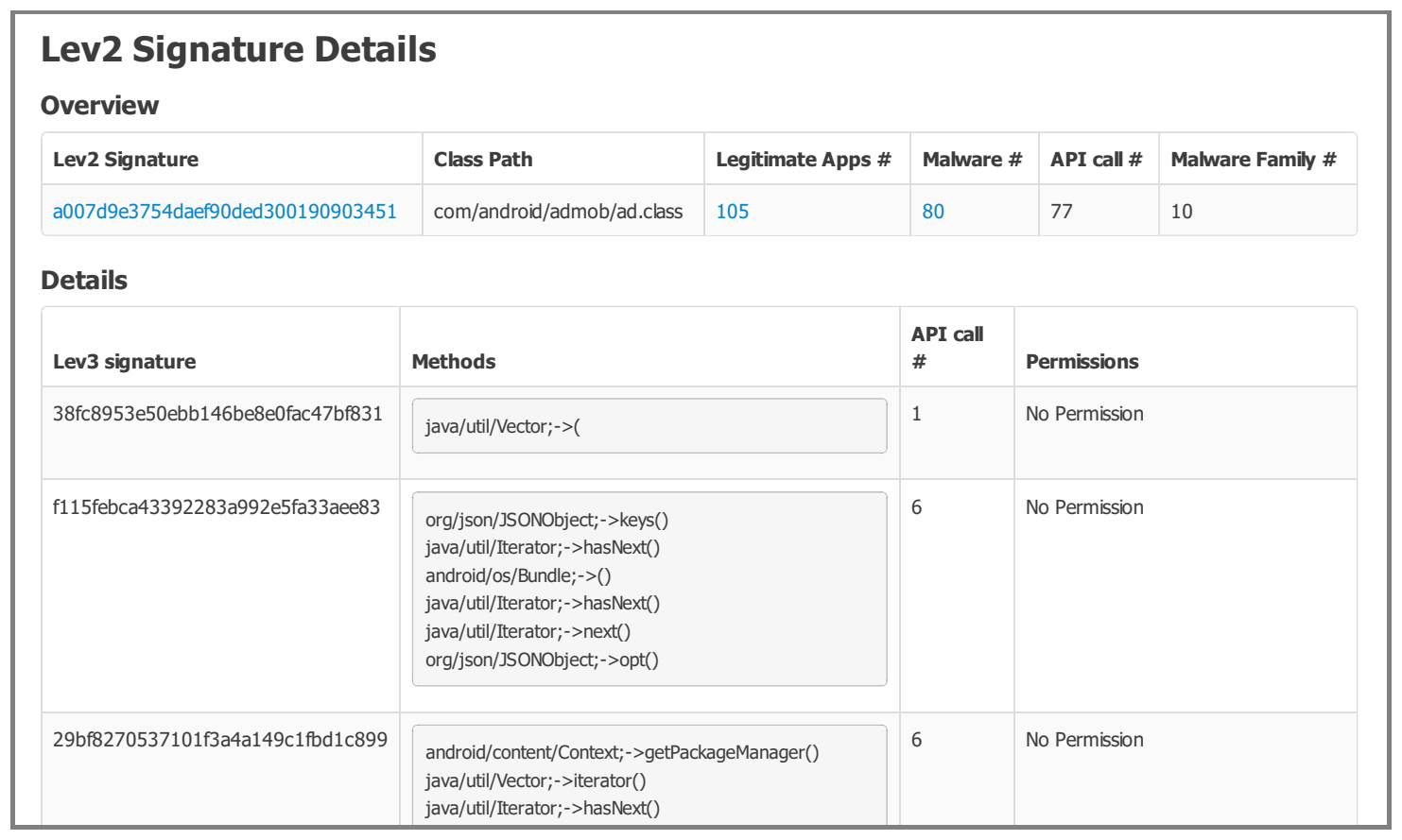

Fig. 10. Detailed Lev2 Signature of Advertisement Library

mon repackaged API calls that correspond to the malicious logic, and ignore the effect of other API calls in these two applications.

Step 3: Assume we have $N$ applications in the database, then we start with $N$ clusters. The distance between two clusters is the similarity score we mentioned in Step 2. We select two applications which have the largest similarity score and combine them into one cluster. For this new cluster, we recalculate the similarity score between this new cluster with other $N-2$ clusters. The new similarity score is computed by averaging all similarity scores between all applications in two different clusters.

Step 4: Again, we combine two clusters which have the largest similarity score. We continue this step until the similarity score between any two clusters is less than a pre-defined threshold $T$ (say $T$ is 100).

Step 5: After we finish the clustering process, we will use anti-virus engines to scan all of these $N$ applications. Each application may be classified as legitimate or malicious.

Step 6: If a cluster has more than $n$ applications (say $n=10$ ) and a small fraction $f$ (say $f \leq 0.2$ ) is classified as malicious. This should be a suspicious cluster since it is very unlikely that more than $n$ applications are similar (in terms of class functionality) in the real-world, and most of them are classified as benign. Hence, the similarity comes when some of these applications are repackaged. We then extract their common classes (using our level 2 signature) and examine these classes. Once we find any malicious logic in these common classes, we discover a zero-day repackaged malware family.

Experimental results in discovering zero-day repackaged malware: Let us present the results of using DroidAnalytics to discover three zero-day repackaged malware families.

- AisRs family: We discover 87 samples of AisRs family in our database. All the malware are repackaged from legitimate applications (e.g., com.fengle.jumptiger, com.mine.videoplayer) and all of them have a common malicious package named "com.ais.rs". This malware contains a number of botnet commands that can be remotely invoked. When the malware runs, it will first communicate with two remote servers (see Figure 11). These two servers are camouflaged as software download websites(see Figure 12). If any of these two servers is online, the malware will receive some commands like downloading other . apk files. They are not necessary malware, but they contain advertisement from the website, 'http://push.aandroid.net'. It is interesting to note that the address is "aandroid.net", not "android.net". Also, one of the many botnet commands is to save the user's application installation lists and system information to the . SQLite file, then upload this file to a remote server.

- AIProvider family: We discover 51 samples of AIProvider family in our database. All the malware are repackaged from legitimate applications (e.g., jinho.eye_check, com.otiasj.androradio) and all of them have a common malicious package named "com.android.internal.provider". There are several interesting characteristics of this malware. Firstly, the malicious package name is disguised as a system package name. Since DroidAnalytics does not detect malware based on the package name, so our system can easily discover this repackaged malware. Secondly, this malware uses DES to encrypt all SMS information (e.g., telephone numbers, SMS content) and store them in the DESUtils class. Thirdly, 


\begin{tabular}{|c|c|c|c|}
\hline Kaspersky & Samples & Antiy & $\overline{\text { Samples }}$ \\
\hline \multirow[t]{3}{*}{ Adrd } & 176 & Adrd & 57 \\
\hline & & AnSer & 5 \\
\hline & & App2card & 8 \\
\hline BaseBrid & 611 & Keji(BaseBrid) & 299 \\
\hline \multirow[t]{2}{*}{ CrWind } & 5 & Crusewin(CrWind) & 5 \\
\hline & & Deduction & 19 \\
\hline \multirow[t]{2}{*}{ DorDrae } & 10 & & \\
\hline & & emagsoftware & 15 \\
\hline FakePlayer & 6 & FakePlayer & 6 \\
\hline Fatakr & 16 & & \\
\hline Fjcon & 142 & fjRece(Fjcon) & 141 \\
\hline Gapev & 5 & gapp(Gapev) & 4 \\
\hline \multirow[t]{2}{*}{ Geinimi } & 139 & Geinimi & 128 \\
\hline & & GingerBreak & 11 \\
\hline GinMaster & 31 & GingerMaster(GinMaster) & 26 \\
\hline Glodream & 13 & GoldDream & 7 \\
\hline \multirow[t]{4}{*}{ Gonca } & 5 & & \\
\hline & & i22hk & 5 \\
\hline & & Itfunz & 10 \\
\hline & & jxtheme & 13 \\
\hline Kmin & 192 & Kmin & 179 \\
\hline \multirow{2}{*}{ KungFu } & 144 & KungFu & 78 \\
\hline & & Lightdd & 7 \\
\hline \multirow[t]{2}{*}{ Lotoor } & 113 & Lotoor & 15 \\
\hline & & MainService & 121 \\
\hline MobileTx & 15 & tianxia(MobileTx) & 14 \\
\hline Nyleaker & 7 & & \\
\hline Opfake & 8 & & \\
\hline Plangton & 126 & Plankton(Plangton) & 1 \\
\hline \multirow[t]{2}{*}{ Rooter } & 26 & DroidDream(Rooter) & 17 \\
\hline & & RootSystemTools & 7 \\
\hline SeaWeth & 11 & seaweed(SeaWeth) & 11 \\
\hline SendPay & 9 & go108(SendPay) & 10 \\
\hline SerBG & 23 & Bgserv(SerBG) & 31 \\
\hline \multirow[t]{5}{*}{ Stiniter } & 50 & & \\
\hline & & Universalandroot & 27 \\
\hline & & Latency & 28 \\
\hline & & Visionaryplus & 1 \\
\hline & & Wukong & 10 \\
\hline \multirow[t]{2}{*}{ Xsider } & 14 & jSMSHider(Xsider) & 8 \\
\hline & & YouBobmg & 20 \\
\hline \multirow[t]{3}{*}{ Yzhc } & 35 & & \\
\hline & & Z4root & 47 \\
\hline & & $\mathrm{Zft}$ & 5 \\
\hline Others (42 families) & 71 & Others (27 families) & 44 \\
\hline Common & 1295 & Common & 1295 \\
\hline All & 2003 & All & 1440 \\
\hline
\end{tabular}

TABLE VI

Detection Results of OUR Cloud-BASEd APK SCANNER

\begin{tabular}{|l|l|l|}
\hline \hline Family Name & Number & Malicious Package Name \\
\hline AisRs & 87 & com.ais.rs \\
Aseiei & 64 & com.aseiei \\
AIProvider & 51 & com.android.internal.provider \\
G3app & 96 & com.g3app \\
GSmstracker & 10 & com.gizmoquip.smstracker \\
YFontmaster & 34 & com.yy.fontmaster \\
\hline All & 342 & \\
\hline \hline
\end{tabular}

TABLE VII

ZERO-DAY REPACKAGED MALWARE SAMPLES

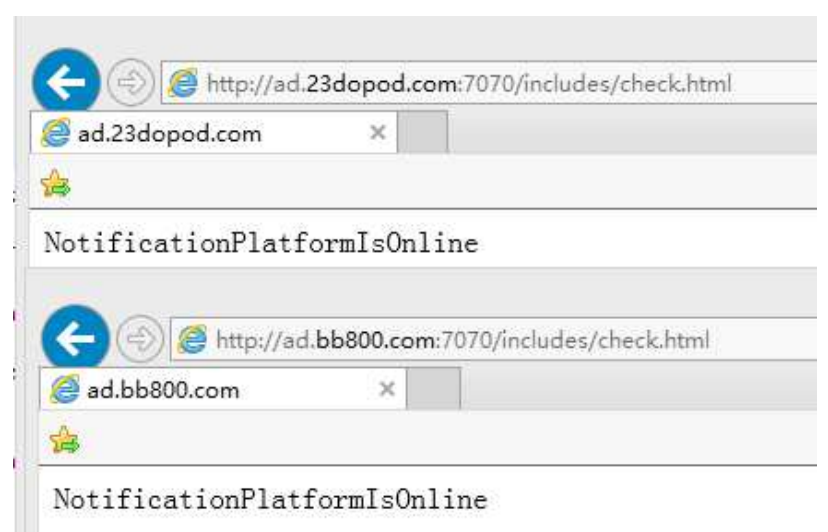

Fig. 11. Remote Servers of AisRs

the malware will start a service called OperateService in the background when it receives "BOOT_COMPLETED" broadcast(see Figure 13 and 14). This service will decrypt the SMS information in the DESUtils class and use this information to send SMS messages without any notification.

- G3app family: We discover 96 samples of G3app family in our database. All the malware are repackaged from legitimate applications (e.g., com.openg3.virtua lIncomingCall,

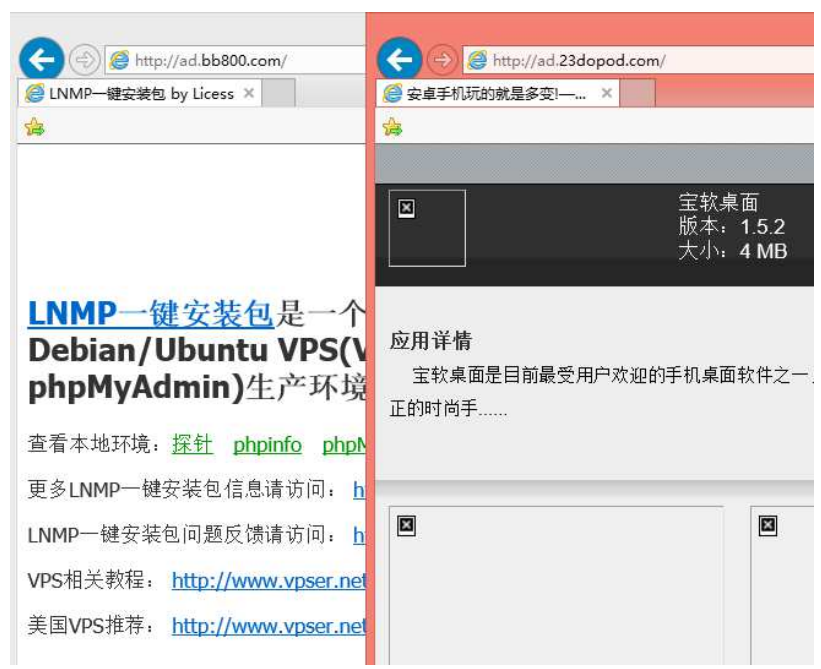

Fig. 12. Camouflaged Software Download Websites 


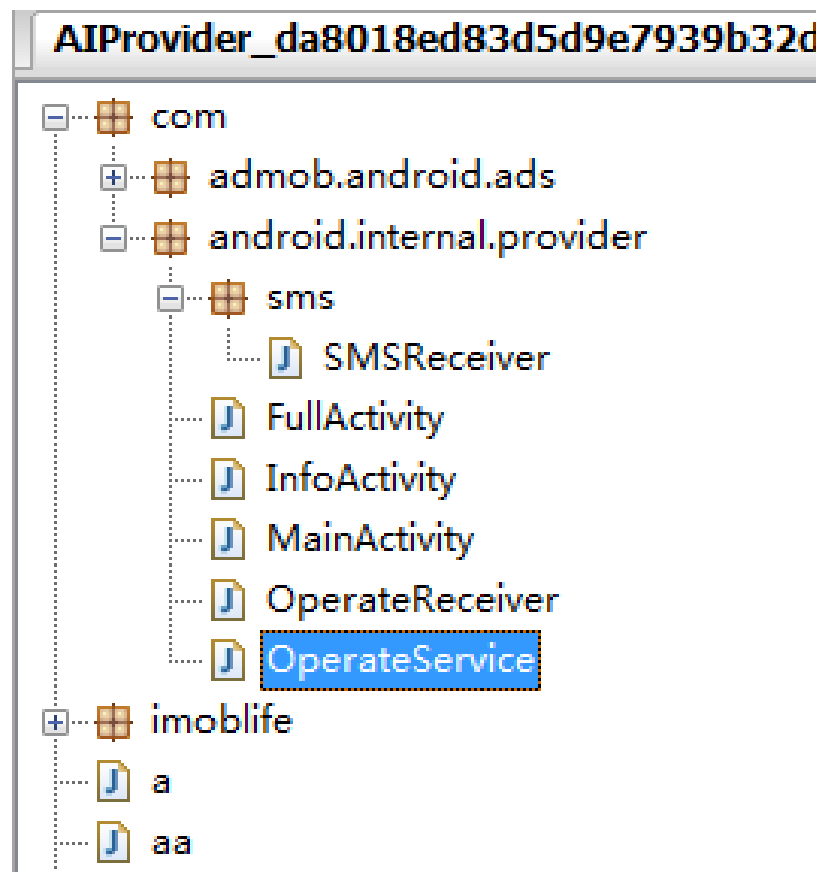

Fig. 13. Disassembled Repackaged Codes of AIProvider

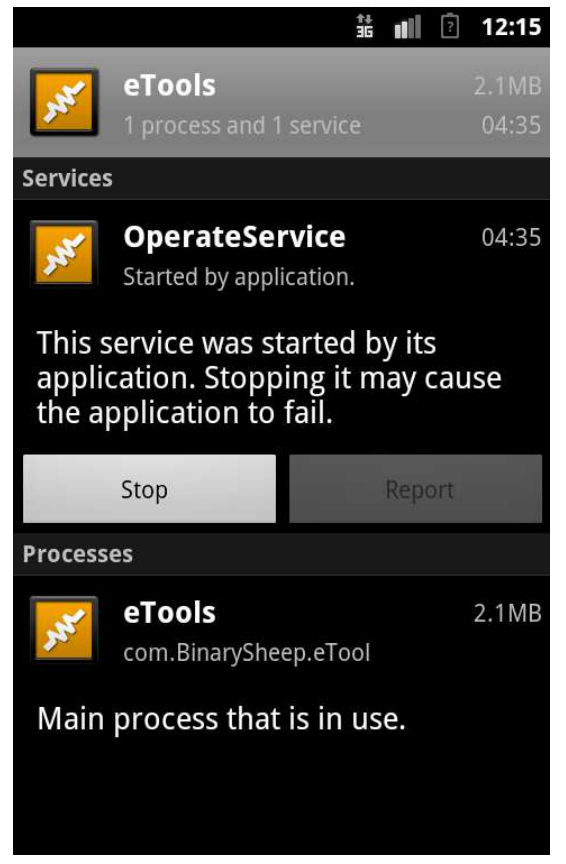

Fig. 14. Malicious OperateService of AIProvider

com.cs.android.wc3) and all of them have a common malicious package named "com.g3app". There are several malicious behaviors in the G3app malware family. Firstly, the malware will frequently pop up notification on the status bar and entice users to select it (see Figure 15). Secondly, the malware will inject trigger codes to every button of the legitimate application(see Figure 16). If the user presses any button in the repackaged application or the notification in the status bar, the malware will download other applications from the remote server. We believe the hackers want to use repackaged malware to publicize their applications and use these advertisements for financial gain.

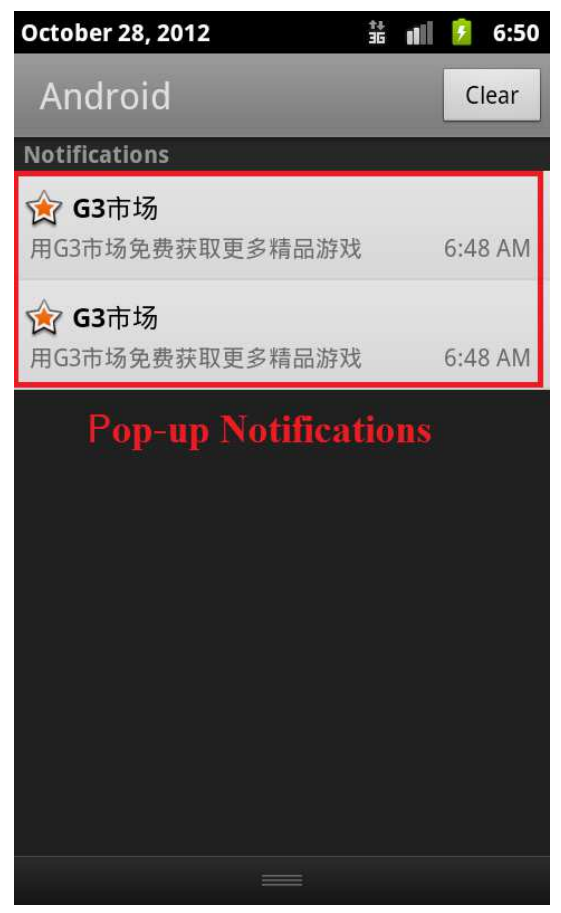

Fig. 15. Trigger Notification of G3app

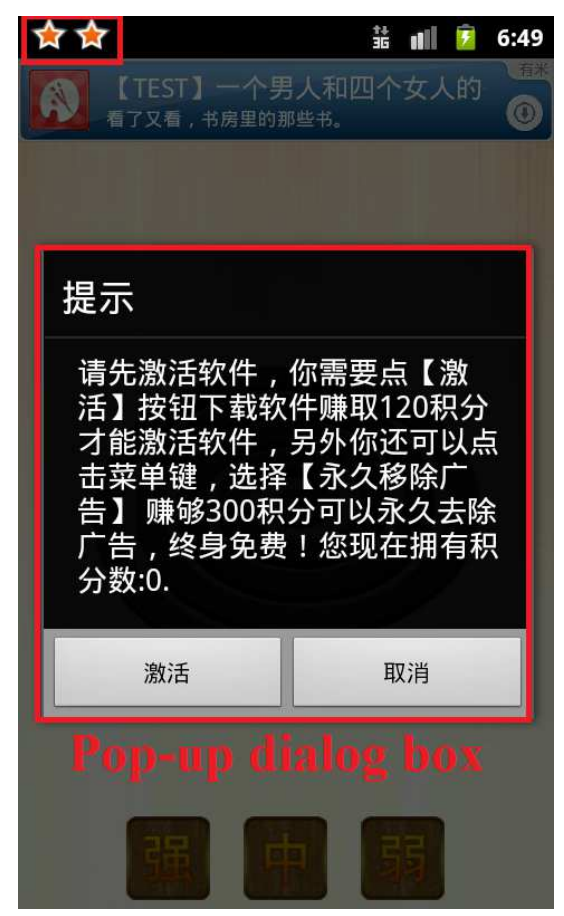

Fig. 16. Trigger Button of G3app 


\section{Related Work}

Before the rapid increase of Android malware in 2011, researchers focused on the permission and capability leaks of Android applications. E.g., David Barrera et al. [23] propose a methodology to explore and analyze permission-based models in Android. Stowaway [16] is a tool for detecting permission over privilege in Android, while ComDroid [24] is a tool which detects communication vulnerabilities. Woodpecker [25] analyzes each application on a smartphone to explore the reachability of a dangerous permission from a public, unguarded interface. William Enck et al. [26] propose a lightweight mobile phone application with a certificationbased permission. In this paper, instead of malware detection, we focus on designing an analytic system that helps analysts to dissect, analyze and correlate Android malware with other Android applications in the database. We propose a novel signature system to identify malicious code segments and associate with other malware in the database. Our signature system is robust against obfuscation techniques that hackers may use.

In August 2010, Kaspersky reported the first SMS Trojan, known as FakePlayer in Android systems [27]. Since then, many malware and their variants have been discovered and mobile malware rapidly became a serious threat. Felt et al. study 18 Android malware in [28]. Enck et al. [29] carry out a study with 1,100 android applications but no malware was found. Zhou Yang et al. [30] study characterization and evolution of Android malware with 1,260 samples. However, they did not show how to systematically collect, analyze and correlate these samples.

Yajin Zhou et al. [31] were the first to present a systematic study for the detection of malicious applications on Android Markets. They successfully discover 211 malware. Their system, DroidRanger, needs malware samples to extract the footprint of each malware family before the known malware detection. For zero day malware, DroidRanger serves as a filtering system. After the filtering process, suspicious malware needs to be manually analyzed. DroidMOSS [32] is an Android system to detect repackaged applications using fuzzy hashing. As stated in [32], the system is not designed for general malware detection. Furthermore, the similarity score provided by DoridMoss is not helpful in malware analysis. In addition, obfuscation techniques can change the order of classes and methods execution, and this will introduce large deviation in the measure used in DroidMOSS. Michael Grace et al. develop RiskRanker [33] to analyze whether a particular application exhibits dangerous behavior. It uses class path as the malware family feature to detect more mutations. However, obfuscation can easily rearrange the opcode along an execution path. So using class path for malware feature is not effective under obfuscation attack. Our system overcomes these problems by using a novel signature algorithm to extract the malware feature at the opcode level so it captures the semantic meaning for signature generation.

For PC based malware, a lot of research work focus on the signature based malware detection. For example, authors in [14] discussed the limitation of using signature to detect malware. In [34], authors described the obfuscation techniques to hide malware. Authors in [35] presented an automatic system to mine specifications of malicious behavior in malware families. Paolo Milani Comparetti et al. [36] proposed a solution to determine malicious functionalities of malware. However, mobile malware has different features as compared with PC based malware. It is difficult to transform a PC based malware detection solution for mobile devices. For example, [3] reported that many anti-virus products have poor performance in detecting Android malware mutations, although these products performed reasonably well for PC based malware. Repackaging is another characteristic of android malware. Authors in [32] showed there are many repackaged applications in Android third party markets and significant number of these applications is malware. Based on the above studies, it is clear that a more sophisticated methodology to detect and analyze Android malware is needed.

\section{Conclusion}

We present DroidAnalytics, an Android malware analytic system which can automatically collect malware, generate signatures for applications, identify malicious code segment (even at the opcode level), and at the same time, associate the malware under study with various malware and applications in the database. Our signature methodology provides significant advantages over traditional cryptographic hash like MD5based signature. We show how to use DroidAnalytics to quickly retrieve, associate and reveal malicious logics. Using the permission recursion technique and class association, we show how to retrieve the permissions of methods, classes and application (rather than basic package information), and associate all applications in the opcode level. Using DroidAnalytics, one can easily discover repackaged applications via the similarity score. Last but not least, we have used DroidAnalytics to detect 2,494 malware samples from 102 families, with 342 zero-day malware samples from six different families. We have conducted extensive experiments to demonstrate the analytic and malware detection capabilities of DroidAnalytics.

\section{REFERENCES}

[1] McAfee, "McAfee Threats Report: Fourth Quarter 2011," Tech. Rep., 2012.

[2] Contagio Mobile. [Online]. Available: http://contagiominidump.blogspot.com/

[3] M. Zheng, P. P. C. Lee, and J. C. S. Lui, "ADAM: An Automatic and Extensible Platform to Stress Test Android Anti-Virus Systems," in Proceedings of the 9th Conference on DIMVA, 2012.

[4] Scrapy. [Online]. Available: http://www.scrapy.org

[5] A. P. Felt, H. Wang, A. Moshchuk, S. Hanna, and E. Chin, "Permission Re-Delegation: Attacks and Defenses," in Proceedings of the 20th USENIX conference on Security, 2011.

[6] G. McCluskey. (1998) Using Java Reflection. [Online]. Available: http://docs.oracle.com/javase/tutorial/reflect/index.html

[7] Google Inc. Google Play Store. [Online]. Available: https://play.google.com/store

[8] AndroidPIT. [Online]. Available: http://www.androidpit.ru

[9] AppChina. [Online]. Available: http://www.appchina.com/

[10] Android App Download Website: SouApp. [Online]. Available: http://www.souapp.com/ 
[11] Mobile Malware Forum. [Online]. Available: https://groups.google.com/forum/?fromgroups=\#!forum/mobilemalware

[12] Android Security Discuss Forum. [Online]. Available: https://groups.google.com/forum/?fromgroups=\#!forum/android-security-discuss

[13] Apktool. [Online]. Available: http://code.google.com/p/android-apktool//

[14] A. Moser, C. Kruegel, and E. Kirda, "Limits of Static Analysis for Malware Detection," in Proceedings of the 23rd Annual Computer Security Applications Conference, 2007.

[15] M. Zheng, P. P. C. Lee, and J. C. S. Lui. ADAM. [Online]. Available: http://ansrlab.cse.cuhk.edu.hk/software/adam/

[16] A. P. Felt, E. Chin, S. Hanna, D. Song, and D. Wagner, "Android Permissions Demystified," in Proceedings of the 18th ACM conference on Computer and communications security, 2011.

[17] DOMOB. [Online]. Available: http://www.domob.cn

[18] AdMob. [Online]. Available: http://www.google.com/ads/admob

[19] Antiy Labs. [Online]. Available: http://www.antiy.net/en/index.html

[20] Virustotal. [Online]. Available: http://www.virustotal.com

[21] Abhi, "Android Malware Injected through Repackaging of Apps," Tech. Rep., 2012.

[22] G. Sims, "Android Malware Genome Project shows that $86 \%$ of all malware delivered via repackaging of legitimate apps," Tech. Rep., 2012.

[23] D. Barrera, H. G. Kayacik, P. C. van Oorschot, and A. Somayaji, "A Methodology for Empirical Analysis of Permission-based Security Models and its Application to Android," in Proceedings of the 17th ACM conference on Computer and communications security, 2010.

[24] E. Chin, A. P. Felt, K. Greenwood, and D. Wagner, "Analyzing Interapplication Communication in Android," in Proceedings of the 9th international conference on Mobile systems, applications, and services, 2011.

[25] M. Grace, Y. Zhou, Z. Wang, and X. Jiang, "Systematic Detection of Capability Leaks in Stock Android Smartphones," in Proceedings of the 19th Annual Network \& Distributed System Security Symposium, 2012.

[26] W. Enck, M. Ongtang, and P. McDaniel, "On Lightweight Mobile Phone Application Certification," in Proceedings of the 16th ACM conference on Computer and communications security, 2009.

[27] C. A. Castillo, "Android Malware Past, Present, and Future," White Paper of McAfee Mobile Security Working Group, 2011.

[28] A. P. Felt, M. Finifter, E. Chin, S. Hanna, and D. Wagner, "A Survey of Mobile Malware in the Wild," in Proceedings of the 1st ACM workshop on Security and privacy in smartphones and mobile devices, 2011.

[29] W. Enck, D. Octeau, P. McDaniel, and S. Chaudhuri, "A Study of Android Application Security," in Proceedings of the 20th USENIX conference on Security, 2011.

[30] Y. Zhou and X. Jiang, "Dissecting Android Malware: Characterization and Evolution," in Proceedings of the 33rd IEEE Symposium on Security and Privacy, 2012.

[31] Y. Zhou, Z. Wang, W. Zhou, and X. Jiang, "Hey, You, Get Off of My Market: Detecting Malicious Apps in Official and Alternative Android Markets," in Proceedings of the 19th Annual Network \& Distributed System Security Symposium, 2012.

[32] W. Zhou, Y. Zhou, X. Jiang, and P. Ning, "DroidMOSS: Detecting Repackaged Smartphone Applications in Third-Party Android Marketplaces," in Proceedings of the second ACM conference on Data and Application Security and Privacy, 2012.

[33] M. Grace, Y. Zhou, Q. Zhang, S. Zou, and X. Jiang, "RiskRanker: Scalable and Accurate Zero-day Android Malware Detection," in Proceedings of the 10th international conference on Mobile systems, applications, and services, 2012.

[34] P. OKane, S. Sezer, and K. McLaughlin, "Obfuscation: The Hidden Malware," IEEE Security and Privacy, 2011.

[35] M. Christodorescu, C. Kruegel, and S. Jha, "Mining Specifications of Malicious Behavior," in Proceedings of the the 6th joint meeting of the European software engineering conference and the ACM SIGSOFT symposium on The foundations of software engineering, 2007.

[36] P. M. Comparetti, G. Salvaneschi, E. Kirda, C. Kolbitsch, C. Kruegel, and S. Zanero, "Identifying Dormant Functionality in Malware Programs," in Proceedings of the 31st IEEE Symposium on Security and Privacy, 2010. 